\title{
2-D Analytical Model for Predicting Magnetic Flux Distribution in Slotless Single-sided Axial Flux Permanent-Magnet Synchronous Machines
}

\author{
A. Ghaffari \\ Department of Electrical and Electronics Engineering, Shiraz University of Technology, Shiraz, I.R. Iran \\ E-mail: a.ghaffari@sutech.ac.ir
}

Received: 25 September 2019; Accepted: 22 October 2019; Available online: 30 November 2019

\begin{abstract}
This paper estimates the magnetic flux density components in the slotless single-sided axial flux permanent-magnet synchronous machines (SAFPMSMs). For this purpose, a 2-D analytical model based on the sub-domain method is utilized in which the cross-section of the presented machine is divided into the seven subregions such as stator side exterior, stator, winding, air-gap, permanent-magnets (PMs), mover and mover side exterior. Based on the Maxwell equations, the related partial differential equations (PDEs) of magnetic flux density components are formed in each sub-region which are identified as the essential step for obtaining the machines quantities. According to the superposition theorem, two separate steps are implemented for calculating the magnetic flux density components. In the first step, open circuit analysis includes various type of magnetization patterns, i.e. parallel, ideal Halbach, 2-segment Halbach and bar magnet in shifting direction is investigated and armature currents are zero and in the second step PMs are inactive and the magnetic flux density components are originated due to only armature reaction. Eventually, 2-D finite element method (FEM) is determined to confirm the accuracy of the presented analytical approach and an acceptable agreement between the analytical and FEM models can be observed.
\end{abstract}

Keywords: Analytical model; Axial flux motor; Magnetic flux density; Maxwell equations; Halbach magnetization.

\section{Introduction}

The increased demand for electric machines and their significant applications has motivated widespread research to improve their performance. Permanent-magnet machines (PMs) and their characteristic features such as high power density and efficiency due to ohmic loss reduction have become truly remarkable among the electrical machines [1-3]. According to the direction of the magnetic flux, PM- machines are divided into two types: axial flux machines (z-direction flux) and radial flux machines (r-direction flux) [4]. Axial flux permanentmagnet machines (AFPMs) plays a significant role in a wide range of industrial applications for their considerable advantages such as high power-to-weight ratio and having more number of poles cause to widely use of them in compact fans and wind turbines [5]. The necessity for a compactable structure made AFPMs a noticeable candidate in the electric vehicles manufacturing [6]. Axial flux machines include various type of structures and four general structures can mention for them as single stator single rotor (SSR) or single-sided, double-sided with internal rotorexternal stator (AFIR), double-sided with internal stator-external rotor (TORUS) and multi-stator multi-rotor that each type has its advantages and disadvantages [7-8].

Numerical and analytical models in electrical machines are known for analyzing and predicting the machine quantities. The analytical model, if possible, is preferred due to the less computational time which is essential in the optimization goals with too many iteration numbers. Also, the user can realize the machine behavior according to the related analytical expression. Besides, for changing the dimensions in the numerical model it is necessary to reconstruct the simulation graphic file while the analytical model is flexible for changing these parameters [9]. In recent year, several analytical models are developed for electrical machines. A 2-D analytical method for predicting the open circuit field distribution in the air-gap region for internal and external topologies in polar coordinates has provided in [10]. The same study has been investigated for parallel magnetization patterns in [11]. In $[12,13]$ a 3-D analytical magnetic field analysis based on magnetic scalar potential and modified Bessel functions have been prepared. The Quasi-3-D electromagnetic field analysis of AFPMs has reported in [14]. A 3$\mathrm{D}$ analysis is more complex and highly time- consuming particularly in designing structure and the 2-D analysis, if possible, is preferred rather than 3-D models [15]. Analytical modeling for a double-sided AFPM is presented in $[16,17]$. Analytical modeling for calculating the air gap flux density, back-EMF and torque which consider the transition zone between poles for micro-motors is presented in [18]. 
Permeability and magnetization patterns are two main challenges of the previous papers in which for simplifying the analytical equations, the permeability of cores are assumed infinite and the flux density distribution in the cores, which is fundamental quantity for defining the core loss, is not realized or the analyzes are limited to the specific magnetization patterns. For instance, in [19], the authors presented an expression to find the axial field in the AFPMs assuming that the core permeability is infinite. An analytical method to calculate the no-load and the armature reaction field for a single-sided AFPMSG has been studied in [20] which the core permeability is assumed infinite. In [21], the authors investigated the analytical modeling of a double-sided axial flux permanentmagnet synchronous generator (AFPMSG). Because of satisfying the boundary conditions is difficult in the radial direction, they tried to develop the proposed method on an exact 2-D solution for magnetic field calculations to find EMF, cogging torque and electromagnetic torque. The governing equations are based on magnetic vector potential calculation and in the form of $\theta$ and $z$ components. They only analyzed parallel magnetization pattern for PMs. In [22] an analytical prediction of armature reaction field distribution in a slotted PM linear synchronous machine based on sub-domain model has reported that the permeability of the core is investigated infinite and PMs flux density distribution is not considered. PMs and armature reaction field distributions considering parallel magnetization pattern in a single-sided coreless AFPMSG for a direct-coupled wind energy system based on Laplace/Poisson equations in terms of $y$ and $\theta$ components and the same work is prepared in [23]. A novel approach for analytical modeling of a slotted AFPMs is provided in [24] where the permeability of cores is assumed infinite.

The main contribution of this paper is to define an accurate 2-D analytical model in which the finite permeability for iron cores are assumed. Also, various magnetization patterns such as parallel, ideal Halbach, 2-segment Halbach and bar magnets in shifting directions magnetization patterns are investigated to predict the magnetic flux density components originated by PMs and armature reaction in the proposed SAFPMSMs.

This article is organized as follows:

In section 2 the proposed analytical procedure is presented and section 3 includes the analytical and numerical models for the tangential and normal components of magnetic flux density due to armature current and PMs in each sub-region to validate the proposed analytical model. Some conclusions are given in section4.

\section{Proposed method}

Fig.1 (a) shows the 3-D configuration of the proposed SAFPMSM and it is correct to analyze a 2-D model instead of 3-D one due to the symmetry of the flux path throughout the motor under the study. Fig. 1(b) illustrates the determined 2-D cross-section to analyze the magnetic flux density component. It is noted that for solving all Maxwell equations in the proposed machine in the Cartesian coordinates is analyzed and in which $x, y$ and $z$ represent $\theta, z$ and $r$ in polar coordinate, respectively. Utilizing the sub-domain method results in the seven subregion and considering a series of assumptions and the basic electromagnetic Maxwell's equations lead to obtaining the related PDEs of the magnetic vector potential in each sub-region that yield to a set of Laplace and Poisson equations and applying curl on the obtained magnetic vector potential results in achieving the magnetic flux density components in each sub-region. Based on the governing PDEs and a set of boundary conditions, a general and particular solution of magnetic flux density in each sub-region are deduced.

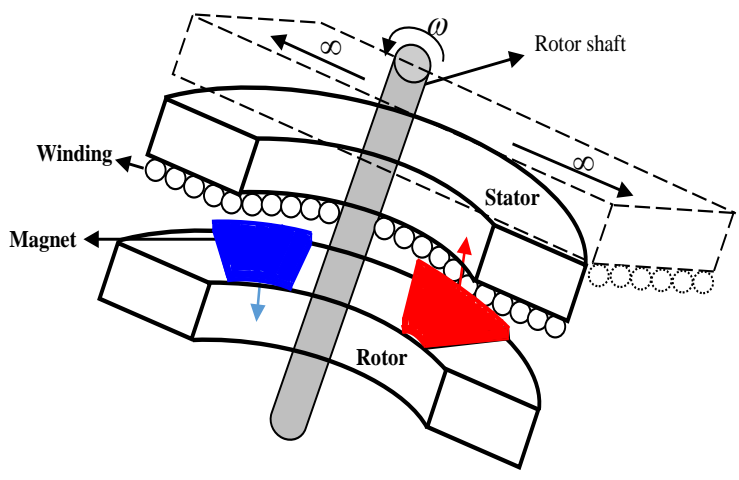

(a) 3-D model

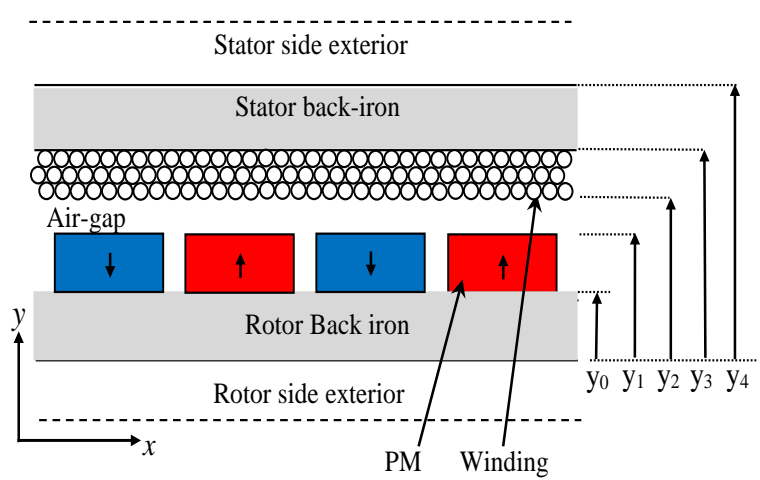

(b) 2-D cross section

Fig. 1. 3-D and 2-D structures of the proposed SAFPMSM

\subsection{The applied assumptions}

By assuming $x, y$ and $z$ instead of $\theta, z$ and $r$ respectively, the following assumptions are formed to present analytical model:

1) The end effects are ignored. 
2) In the case of $2 \mathrm{D}$ problems, it is assumed that $\boldsymbol{A}=\left[0,0, A_{z}(x, y)\right], \boldsymbol{M}=\left[M_{x}(x), M_{y}(x), 0\right]$ and $\boldsymbol{B}=\left[B_{x}(x, y), B_{y}(x, y), 0\right]$ where $\boldsymbol{A}, \boldsymbol{M}$ and $\boldsymbol{B}$ are magnetic vector potential, magnetization pattern and magnetic flux density, respectively.

3) All materials are isotropic.

4) The media have finite relative permeability.

5) The saturation effects are neglected.

6) The motor has a slotless stator structure.

7) The eddy current reaction field is neglected.

\subsection{Governing PDEs}

According to the Maxwell equations the related PDEs in each sub-region of the motor under the study are deduced as follow:

$$
-\nabla^{2} \boldsymbol{A}=\mu_{0} \mu_{r} \boldsymbol{J}+\mu_{0} \nabla \times \boldsymbol{M}
$$

where $\boldsymbol{J}$ is the armature current density vector, that is zero in the case of open-circuit field calculations and $\boldsymbol{M}$ is magnetization vector which is zero for obtaining magnetic flux density components due to armature reaction.

\subsection{Magnetic flux density originated by PMs}

To obtain the magnetic flux density originated due to PMs, armature currents must be zero $(\boldsymbol{J}=0)$ and two various kind of PDEs are obtained to estimating the magnetic flux density components in all sub-regions. The first category includes rotor-side exterior (er), air-gap (a), winding (w), stator (s) and stator-side exterior (es) that the related PDEs are Laplace equations as follow:

$\nabla^{2} A^{i}=0 \quad i=\{e r, r, a, w, s, e s\}$

The second PDEs is Poisson one that allocated to PM sub-region and describe as follow:

$\nabla^{2} \boldsymbol{A}^{P M}=-\mu_{0} \nabla \times \boldsymbol{M}$

where $\mu_{0}$ is free space permeability and $\boldsymbol{M}$ is defined as its Fourier series expansions of the tangential and normal components of each magnetization patterns as:

$$
\begin{aligned}
& \boldsymbol{M}=M_{x} a_{x}+M_{y} a_{y} \\
& M_{x}(x)=\sum_{n=1}^{\infty} m_{x n} \cos \left(\alpha_{n} x\right) \\
& M_{y}(\theta)=\sum_{n=1}^{\infty} m_{y n} \sin \left(\alpha_{n} x\right)
\end{aligned}
$$

where $\alpha_{n}=n \pi / \tau_{p}$ in which $\tau_{p}$ is pole pitch, $m_{x n}$ and $m_{y n}$ are Fourier series components and these components, as well as magnetization patterns, are shown in Fig. 2.

Utilizing separation variable method in (2) and (3) leads to obtain the following expression for the magnetic vector potential in two illustrated sub-regions:

$$
\begin{aligned}
& A_{z}^{i}(x, y)=\sum_{n=1}^{\infty}\left(a_{n}^{i} \sinh \left(\alpha_{n} y\right)+b_{n}^{i} \cosh \left(\alpha_{n} y\right)\right) \cos \left(\alpha_{n} x\right)+\left(c_{n}^{i} \sinh \left(\alpha_{n} y\right)+d_{n}^{i} \cosh \left(\alpha_{n} y\right)\right) \sin \left(\alpha_{n} x\right) \\
& A_{z}^{P M}(x, y)=\sum_{n=1}^{\infty}\left(a_{n}^{P M} \sinh \left(\alpha_{n} y\right)+b_{n}^{P M} \cosh \left(\alpha_{n} y\right)+\frac{\mu_{0}}{\alpha_{n}} m_{x n}\right) \cos \left(\alpha_{n} x\right)+\left(c_{n}^{P M} \sinh \left(\alpha_{n} y\right)+\right. \\
& \left.d_{n}^{P M} \cosh \left(\alpha_{n} y\right)-\frac{\mu_{0}}{\alpha_{n}} m_{y n}\right) \sin \left(\alpha_{n} x\right)
\end{aligned}
$$

Magnetic flux density components in each sub-regions can easily deduce by applying curl on the obtained magnetic vector potential and the tangential and normal components of magnetic flux density can define as:

$$
\begin{aligned}
& B_{x}=\frac{\partial A_{z}}{\partial y} \\
& B_{y}=-\frac{\partial A_{z}}{\partial x}
\end{aligned}
$$


It is noted that for showing the rotor motion $x$ must be substituted by $x-d$ which $d$ is the rotor motion and illustrated as:

$$
d=v t+d_{0}
$$

where $v$ is converted rotor angular velocity $(\omega)$ to the linear translation speed, $t$ is time and $d_{0}$ is initial rotor position.

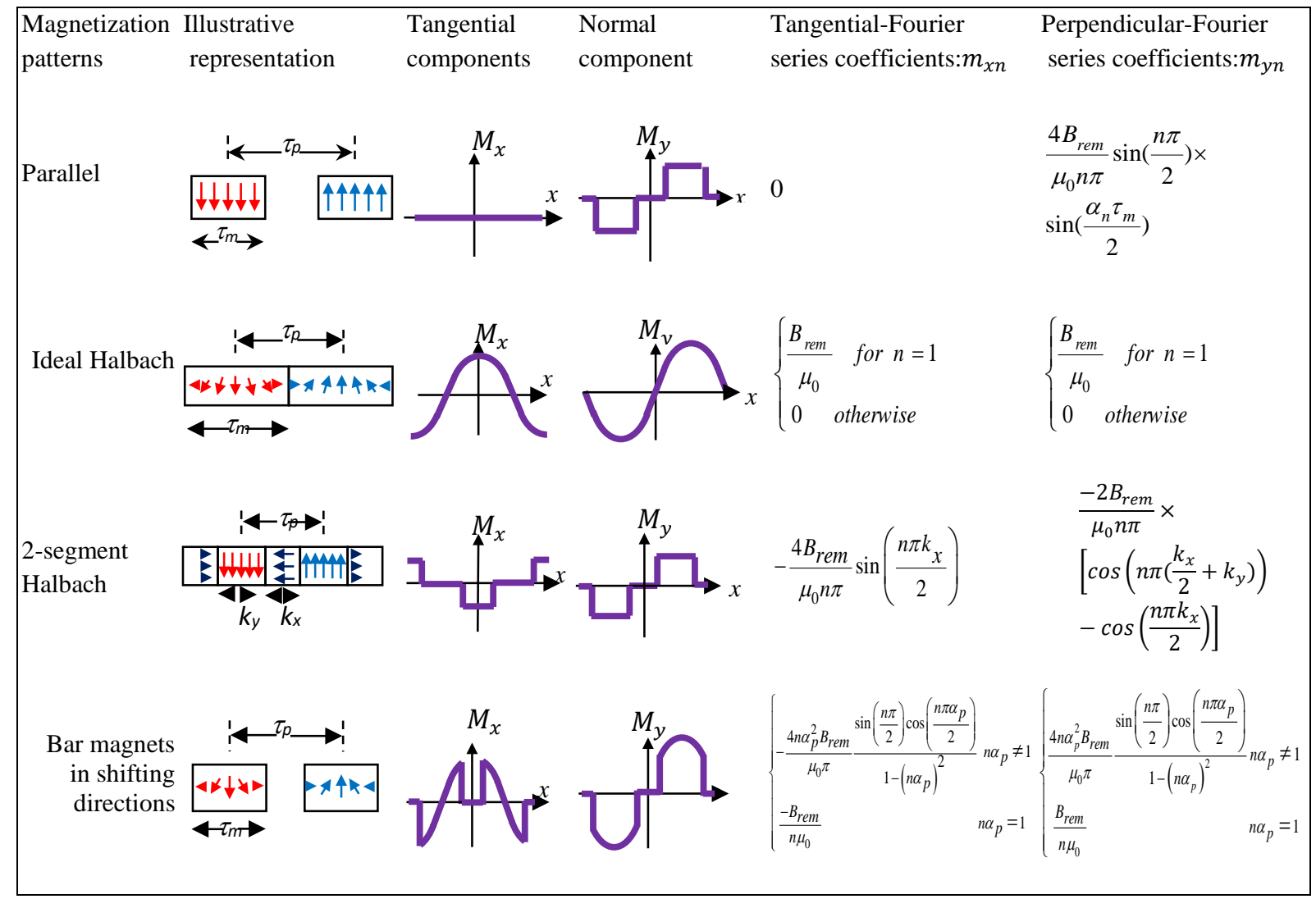

${ }^{*} k_{\theta}$ and $k_{z}$ are the $\theta$-direction magnetized PM width to the pole pitch ratio and the $z$-direction magnetized PM width to the pole pitch ratio, Respectively. $B_{r e m}$ is the permanent-magnet remanence flux density, $\tau_{m}$ is the magnet width and $\alpha_{p}=\tau_{m} / \tau_{p}$.

Fig. 2. Magnetization patterns and Fourier series components for the proposed SAFPMSM

\subsection{Magnetic flux density originated by armature currents}

To obtain magnetic flux density originated by armature reaction PMs must be inactive $(\boldsymbol{M}=0)$. According to the Laplace and Poison equations, similar to the last section, two categories can define for obtaining the related magnetic vector potential in each sub-region. The first group includes rotor-side exterior, rotor, PMs, air-gap, stator and stator-side exterior in which the PDEs in these regions are Laplace one and PDEs for them similar to (2) is realized as:

$$
\nabla^{2} A^{i}=0 \quad i=\{e r, r, P M s, a, s, e s\}
$$

These PDEs in the winding sub-regions is Poison one and extracted as:

$$
\nabla^{2} \boldsymbol{A}^{w}=-\mu_{0} \boldsymbol{J}
$$

Like magnetization patterns, the current density is introduced by its Fourier series expansion as follow:

$$
J(x, t)=\sum_{n=1}^{\infty} J_{1 n} \sin \left(\alpha_{n} x\right)+J_{2 n} \cos \left(\alpha_{n} x\right)
$$

where $J_{1 n}$ and $J_{2 n}$ are Furrier series components of current density. By exciting three sinusoidal currents into the winding coils the current density Furrier components are calculated as: 


$$
\begin{aligned}
& J_{1 n}=-\frac{2 N_{t} \tau_{p}}{3\left(y_{2}-y_{3}\right)} \frac{\cos \left(\frac{2 n \pi}{3}\right)-\cos \left(\frac{n \pi}{3}\right)}{n \pi} \times\left(i_{a}+i_{b} \cos \left(\frac{2 n \pi}{3}\right)+i_{c} \cos \left(\frac{2 n \pi}{3}\right)\right) \\
& J_{2 n}=-\frac{2 N_{t} \tau_{p}}{3\left(y_{2}-y_{3}\right)} \frac{\cos \left(\frac{2 n \pi}{3}\right)-\cos \left(\frac{n \pi}{3}\right)}{n \pi} \times\left(-i_{b} \sin \left(\frac{2 n \pi}{3}\right)+i_{c} \sin \left(\frac{2 n \pi}{3}\right)\right) .
\end{aligned}
$$

where $N_{t}$ is the number of turns for each coil and applied current in the proposed machine are defined as:

$$
\begin{aligned}
& i_{a}(t)=I_{m} \sin (\omega t) \\
& i_{b}(t)=I_{m} \sin \left(\omega t-\frac{2 \pi}{3}\right) \\
& i_{c}(t)=I_{m} \sin \left(\omega t+\frac{2 \pi}{3}\right)
\end{aligned}
$$

where $I_{m}$ is the maximum phase current. Substituting current density Furrier series expansion in (14) and solving PDEs in each sub-region leads to obtain the following equation for magnetic vector potential in each sub-region due to only armature current:

$$
\begin{aligned}
A_{z}^{i}(x, y)= & \sum_{n=1}^{\infty}\left(a_{n}^{i} \sinh \left(\alpha_{n} y\right)+b_{n}^{i} \cosh \left(\alpha_{n} y\right)\right) \cos \left(\alpha_{n} x\right)+\left(c_{n}^{i} \sinh \left(\alpha_{n} y\right)+d_{n}^{i} \cosh \left(\alpha_{n} y\right)\right) \sin \left(\alpha_{n} x\right) \\
A_{z}^{w}(x, y)= & \sum_{n=1}^{\infty}\left(a_{n}^{w} \sinh \left(\alpha_{n} y\right)+b_{n}^{w} \cosh \left(\alpha_{n} y\right)+\frac{\mu_{0}}{\alpha_{n}^{2}} J_{2 n}\right) \cos \left(\alpha_{n} x\right)+\left(c_{n}^{w} \sinh \left(\alpha_{n} y\right)+\right. \\
& \left.d_{n}^{w} \cosh \left(\alpha_{n} y\right)+\frac{\mu_{0}}{\alpha_{n}^{2}} J_{1 n}\right) \sin \left(\alpha_{n} x\right)
\end{aligned}
$$

Similar to the previous section, applying curl on the obtained magnetic vector potential leads to determine the magnetic flux density components.

\subsection{The boundary conditions}

By obtaining PDEs, the magnetic potential coefficients in each equation of the proposed sub-regions remain unknown. On the other hand, the aforementioned PDEs need to satisfy the boundary conditions at the interface between two adjacent sub-regions. In the presented axial flux motor, the boundary conditions at each interface can be written as:

$$
\begin{aligned}
& \left.H_{x}^{i}(x, y)\right|_{y=Y}=\left.H_{x}^{i+}(x, y)\right|_{y=Y} \\
& \left.B_{y}^{i}(x, y)\right|_{y=Y}=\left.B_{y}^{i+}(x, y)\right|_{y=Y}
\end{aligned}
$$

where $i$ and $i^{+}$represent two adjacent sub-regions and these boundary conditions can be formed as:

$$
\left(i, i^{+}, Y\right)=\left\{(e r, r, 0),\left(r, p m, y_{0}\right),\left(p m, a, y_{1}\right),\left(a, w, y_{2}\right),\left(w, s, y_{3}\right),\left(s, e s, y_{4}\right)\right\}
$$

To have limited results of the magnetic flux density components, some of the unknown coefficients must be zero like $b_{n}^{e r}=d_{n}^{e r}=a_{n}^{e s}=b_{n}^{e s}=0$. Therefore, 24 unknown coefficients such as $a_{n}^{e r}, c_{n}^{, e r}, a_{n}^{r}, b_{n}^{r}$, $c_{n}^{r}, d_{n}^{r}, a_{n}^{P M}, b_{n}^{P M}, c_{n}^{P M}, d_{n}^{P m}, a_{n}^{a}, b_{n}^{a}, c_{n}^{a}, d_{n}^{a}, a_{n}^{w}, b_{n}^{w}, c_{n}^{w}, d_{n}^{w}, a_{n}^{s}, b_{n}^{s}, c_{n}^{s}, d_{n}^{s}, b_{n}^{e s}, d_{n}^{e s}$ are available and 24 independent equations are necessary to predict the magnetic flux density components in each sub-region. Applying the boundary conditions result in forming these 24 equations and all these simultaneous algebraic equations are written in the Appendix.

\section{Case study}

To validate the proposed analytical model, the FEM model is utilized and analytical results of magnetic flux density components are compared with those obtained from FEM. Table 1 lists the specifications of the motor under the study that is applied to reveal the accuracy of the analytical model. Fig. 3 illustrates analytical and numerical results of the normal and tangential components of magnetic flux density in all sub-regions of the proposed axial flux motor due to only four mentioned magnetization patterns and an acceptable agreement between these two models is observable. The interesting point in the three Halbach magnetization patterns is related to the magnetic flux path that it passes manly through the PMs and the magnetic flux in the rotor is negligible and it is possible to replace the rotor with other material having less weight, less volume and cheaper than the ferromagnetic 
materials. Also, it is evident that the ideal Halbach magnetization includes less THD compared with other magnetization patterns.

Table 1. Main design parameters of the proposed axial flux motor under the study

\begin{tabular}{llc}
\hline Parameters & Symbols & Values \\
\hline Rotor back iron height & $y_{0}$ & $8 \mathrm{~mm}$ \\
PM height & $y_{1}-y_{0}$ & $7 \mathrm{~mm}$ \\
Air-gap height & $y_{2}-y_{1}$ & $1.5 \mathrm{~mm}$ \\
Winding height & $y_{3}-y_{2}$ & $8 \mathrm{~mm}$ \\
Stator back iron height & $y_{4}-y_{3}$ & $10 \mathrm{~mm}$ \\
Stator relative permeability & $\mu_{r}^{s}$ & 1500 \\
Mover relative permeability & $\mu_{r}^{r}$ & 1000 \\
PM relative permeability & $\mu_{r}^{p m}$ & 1.04 \\
PM width for the parallel pattern & $\tau_{m}$ & $40 \mathrm{~mm}$ \\
Pole pitch & $\tau_{p}$ & $50 \mathrm{~mm}$ \\
Number of poles & $p$ & 4 \\
X-direction magnetized PM width to the pole pitch ratio for 2-segment Halbach & $k_{x}$ & 0.35 \\
y-direction magnetized PM width to the pole pitch ratio for 2-segment Halbach & $k_{y}$ & 0.65 \\
PM Remanence flux density & $B_{r e m}$ & $1.1 \mathrm{~T}$ \\
Peak armature current & $I_{m}$ & $4 \mathrm{~A}$ \\
Number of turns per coil & $N_{t}$ & 66 \\
Number of coils per phase & $N_{c}$ & 2 \\
Filling factor & $K_{f}$ & 0.5 \\
\hline
\end{tabular}
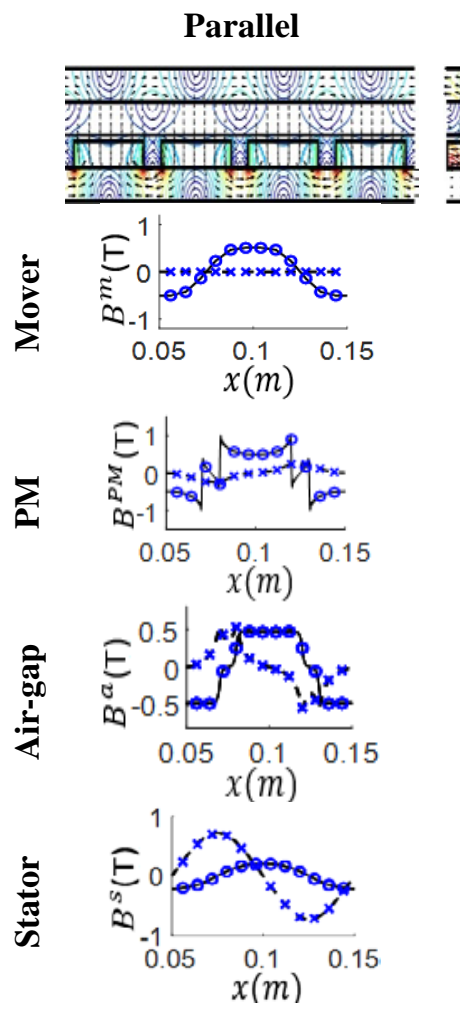

Ideal Halbach
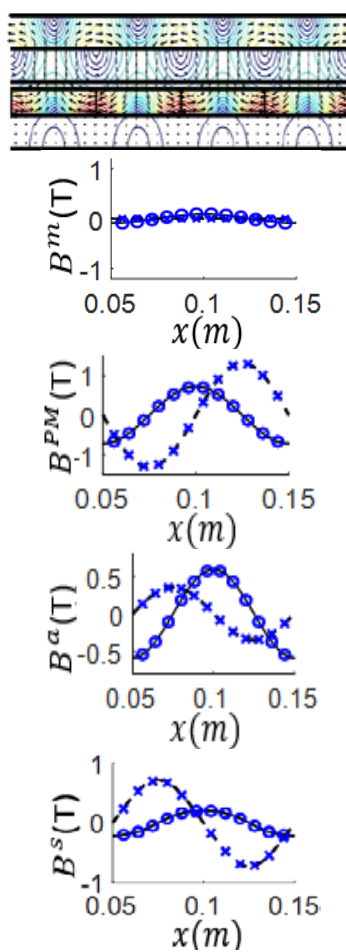

2-segment Halbach
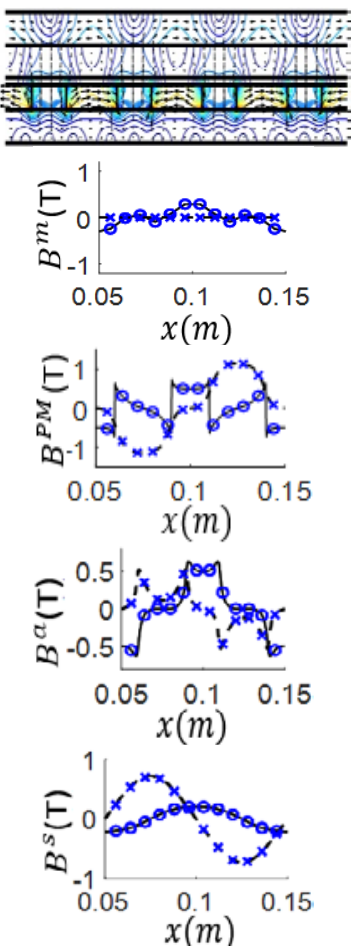

Bar magnets in shifting directions
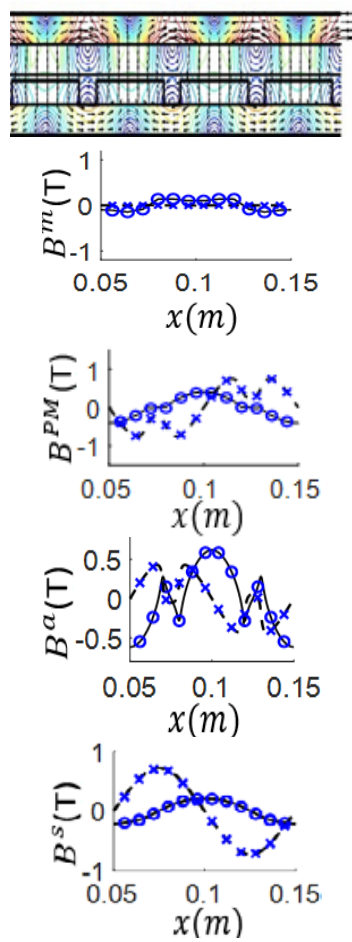

o Numerical results of the normal component of the flux density, $x$ Numerical results of the tangential component of the flux density, - - Analytical results of the tangential components of the flux density, - Analytical results of the normal component of the flux density

Fig. 3. Analytical and numeric results of flux density distribution due to only PMs in proposed SAFPMSM

Another exciting point in the simulation procedure is related to the time of the simulation that for the analytical model is 13 times less than the numerical one. It means in the design stage and optimization problem includes too many iterations, times can be saved by implementing the analytical model, if possible, instead of the numerical 
model. Fig. 4 describes the magnetic flux density distributions due to the only armature reaction current that the slotless structure causes to increase the magnetic air-gap and the magnetic flux density components originated by armature currents are less than these components due to the PMs.





Mover



Air-gap

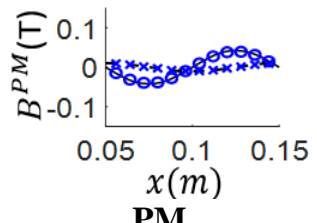

PM

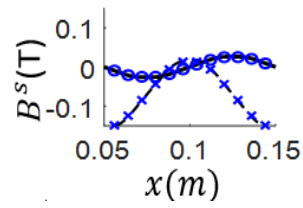

Stator

- - Analytical results of the tangential component of the flux density, - Analytical results of the normal component of the flux density, o Numerical results of the normal component of the flux density, $\times$ Numerical results of the tangential component of the flux density

Fig. 4. Flux density distribution due to only armature current in the motor under the study

\section{Conclusions}

This paper focuses on the analytical magnetic field calculations for the slotless single-sided axial flux permanent-magnet synchronous machines with surface mounted magnets. Sub-domain method is applied due to its accuracy and by this model the effects of armature currents and PMs with different magnetization patterns (i.e. parallel, ideal Halbach, 2-segment and bar magnets in shifting directions patterns) on the magnetic flux density distribution in all sub-regions like rotor, PMs, air-gap, winding and stator are scrutinized. Finally, both PM and armature reaction results are confirmed by the FEM method. Also, less computational time of the analytical model rather than the numerical one is discussed and the extracted results revealed that the computational time of the analytical model was 13 times less than that of numeric.

\section{Appendix}

Imposing the boundary conditions between two adjacent regions and applying the defined symbols in Table 1 lead to the following 24 equations:

$a$ : Interface between rotor-side exterior and rotor at $y=0$

$$
\begin{aligned}
& -\mu_{r}^{r} a_{n}^{e r}-a_{n}^{r}+b_{n}^{r}=0 \\
& -\mu_{r}^{r} c_{n}^{e r}-c_{n}^{r}+d_{n}^{r}=0 \\
& -a_{n}^{e r}+a_{n}^{r}+b_{n}^{r}=0 \\
& -c_{n}^{e r}+c_{n}^{r}+d_{n}^{m}=0
\end{aligned}
$$

$b$ : Interface between rotor and PMs at $y=y_{0}$

$$
\begin{aligned}
& \mu_{r}^{p m} a_{n}^{r} \sinh \left(\alpha_{n} y_{0}\right)-\mu_{r}^{p m} b_{n}^{r} \cosh \left(\alpha_{n} y_{0}\right)-\mu_{r}^{r} a_{n}^{p m} \sinh \left(\alpha_{n} y_{0}\right)+\mu_{r}^{r} b_{n}^{p m} \cosh \left(\alpha_{n} y_{0}\right)=-\frac{\mu_{r}^{r} \mu_{0} m_{X n}}{\alpha_{n}} \cos \left(\alpha_{n} d\right) \\
& \mu_{r}^{p m} c_{n}^{r} \sinh \left(\alpha_{n} y_{0}\right)-\mu_{r}^{p m} d_{n}^{r} \cosh \left(\alpha_{n} y_{0}\right)-\mu_{r}^{r} c_{n}^{p m} \sinh \left(\alpha_{n} y_{0}\right)+\mu_{r}^{r} d_{n}^{p m} \cosh \left(\alpha_{n} y_{0}\right)=-\frac{\mu_{r}^{r} \mu_{0} m_{x n}}{\alpha_{n}} \sin \left(\alpha_{n} d\right) \\
& -a_{n}^{r} \sinh \left(\alpha_{n} y_{0}\right)-b_{n}^{r} \cosh \left(\alpha_{n} y_{0}\right)+a_{n}^{p m} \sinh \left(\alpha_{n} y_{0}\right)-b_{n}^{p m} \cosh \left(\alpha_{n} y_{0}\right)=-\frac{\mu_{0} m_{y n}}{\alpha_{n}} \cos \left(\alpha_{n} d\right) \\
& -c_{n}^{r} \sinh \left(\alpha_{n} y_{0}\right)-d_{n}^{r} \cosh \left(\alpha_{n} y_{0}\right)-c_{n}^{p m} \sinh \left(\alpha_{n} y_{0}\right)-d_{n}^{p m} \cosh \left(\alpha_{n} y_{0}\right)=\frac{\mu_{0} m_{y n}}{\alpha_{n}} \sin \left(\alpha_{n} \delta\right) .
\end{aligned}
$$


c: Interface between PMs and air-gap at $y=y_{1}$

$$
\begin{aligned}
& a_{n}^{p m} \sinh \left(\alpha_{n} y_{1}\right)-b_{n}^{p m} \cosh \left(\alpha_{n} y_{1}\right)-\mu_{r}^{p m} a_{n}^{a} \sinh \left(\alpha_{n} y_{1}\right)+\mu_{r}^{p m} b_{n}^{a} \cosh \left(\alpha_{n} y_{1}\right)=\frac{\mu_{0} m_{x n}}{\alpha_{n}} \cos \left(\alpha_{n} d\right) \\
& c_{n}^{p m} \sinh \left(\alpha_{n} y_{1}\right)-d_{n}^{p m} \cosh \left(\alpha_{n} y_{1}\right)-\mu_{r}^{p m} c_{n}^{a} \sinh \left(\alpha_{n} y_{1}\right)+\mu_{r}^{p m} d_{n}^{a} \cosh \left(\alpha_{n} y_{1}\right)=-\frac{\mu_{0} m_{x n}}{\alpha_{n}} \sin \left(\alpha_{n} d\right) \\
& -a_{n}^{p m} \sinh \left(\alpha_{n} y_{1}\right)-b_{n}^{p m} \cosh \left(\alpha_{n} y_{1}\right)+a_{n}^{a} \sinh \left(\alpha_{n} y_{1}\right)+b_{n}^{a} \cosh \left(\alpha_{n} y_{1}\right)=\frac{\mu_{0} m_{y n}}{\alpha_{n}} \cos \left(\alpha_{n} d\right) \\
& c_{n}^{p m} \sinh \left(\alpha_{n} y_{1}\right)+d_{n}^{p m} \cosh \left(\alpha_{n} y_{1}\right)-c_{n}^{a} \sinh \left(\alpha_{n} y_{1}\right)-d_{n}^{a} \cosh \left(\alpha_{n} y_{1}\right)=-\frac{\mu_{0} m_{y n}}{\alpha_{n}} \sin \left(\alpha_{n} d\right) .
\end{aligned}
$$

$d$ : Interface between air-gap and winding at $y=y_{2}$

$$
\begin{aligned}
& a_{n}^{a} \sinh \left(\alpha_{n} y_{2}\right)-b_{n}^{a} \cosh \left(\alpha_{n} y_{2}\right)-a_{n}^{w} \sinh \left(\alpha_{n} y_{2}\right)+b_{n}^{w} \cosh \left(\alpha_{n} y_{2}\right)=0 \\
& c_{n}^{a} \sinh \left(\alpha_{n} y_{2}\right)-d_{n}^{a} \cosh \left(\alpha_{n} y_{2}\right)-c_{n}^{w} \sinh \left(\alpha_{n} y_{2}\right)+d_{n}^{w} \cosh \left(\alpha_{n} y_{2}\right)=0 \\
& -a_{n}^{a} \sinh \left(\alpha_{n} y_{2}\right)-b_{n}^{a} \cosh \left(\alpha_{n} y_{2}\right)+a_{n}^{w} \sinh \left(\alpha_{n} y_{2}\right)+b_{n}^{w} \cosh \left(\alpha_{n} y_{2}\right)=-\frac{\mu_{0} J_{2 n}}{\alpha_{n}^{2}} \\
& -c_{n}^{a} \sinh \left(\alpha_{n} y_{2}\right)-d_{n}^{a} \cosh \left(\alpha_{n} y_{2}\right)+c_{n}^{w} \sinh \left(\alpha_{n} y_{2}\right)+d_{n}^{w} \cosh \left(\alpha_{n} y_{2}\right)=-\frac{\mu_{0} J_{1 n}}{\alpha_{n}^{2}}
\end{aligned}
$$

$e$ : Interface between winding and stator at $y=y_{3}$

$$
\begin{aligned}
& \mu_{r}^{s} a_{n}^{w} \sinh \left(\alpha_{n} y_{3}\right)-\mu_{r}^{s} b_{n}^{w} \cosh \left(\alpha_{n} y_{3}\right)-a_{n}^{s} \sinh \left(\alpha_{n} y_{3}\right)+b_{n}^{S} \cosh \left(\alpha_{n} y_{3}\right)=0 \\
& \mu_{r}^{S} c_{n}^{w} \sinh \left(\alpha_{n} y_{3}\right)-\mu_{r}^{s} d_{n}^{w} \cosh \left(\alpha_{n} y_{3}\right)-c_{n}^{s} \sinh \left(\alpha_{n} y_{3}\right)+d_{n}^{s} \cosh \left(\alpha_{n} y_{3}\right)=0 \\
& a_{n}^{w} \sinh \left(\alpha_{n} y_{3}\right)+b_{n}^{w} \cosh \left(\alpha_{n} y_{3}\right)-a_{n}^{s} \sinh \left(\alpha_{n} y_{3}\right)-b_{n}^{S} \cosh \left(\alpha_{n} y_{3}\right)=-\frac{-\mu_{0} J_{2 n}}{\alpha_{n}^{2}} \\
& c_{n}^{w} \sinh \left(\alpha_{n} y_{3}\right)+d_{n}^{w} \cosh \left(\alpha_{n} y_{3}\right)-c_{n}^{s} \sinh \left(\alpha_{n} y_{3}\right)-d_{n}^{s} \cosh \left(\alpha_{n} y_{3}\right)=-\frac{-\mu_{0} J_{1 n}}{\alpha_{n}^{2}}
\end{aligned}
$$

$f$ : Interface between stator and stator-side exterior at $y=y_{4}$

$$
\begin{aligned}
& a_{n}^{S} \sinh \left(\alpha_{n} y_{4}\right)-b_{n}^{S} \cosh \left(\alpha_{n} y_{4}\right)+\mu_{r}^{S} b_{n}^{e s} \cosh \left(\alpha_{n} y_{4}\right)=0 \\
& c_{n}^{s} \sinh \left(\alpha_{n} y_{4}\right)-d_{n}^{S} \cosh \left(\alpha_{n} y_{4}\right)+\mu_{r}^{S} d_{n}^{e s} \cosh \left(\alpha_{n} y_{4}\right)=0 \\
& a_{n}^{s} \sinh \left(\alpha_{n} y_{4}\right)+b_{n}^{s} \cosh \left(\alpha_{n} y_{4}\right)-b_{n}^{e s} \cosh \left(\alpha_{n} y_{4}\right)=0 \\
& c_{n}^{S} \sinh \left(\alpha_{n} y_{4}\right)+d_{n}^{S} \cosh \left(\alpha_{n} y_{4}\right)-d_{n}^{S} \cosh \left(\alpha_{n} y_{4}\right)=0
\end{aligned}
$$

\section{References}

[1] Di Gerlando A, Foglia G, Iacchetti MF, Perini R. Axial flux PM machines with concentrated armature windings: Design analysis and test validation of wind energy generators. IEEE Transactions on Industrial Electronics. 2011;58(9):3795-3805.

[2] Curiac P, Kang DH. Preliminary evaluation of a megawatt-class low-speed axial flux PMSM with selfmagnetization function of the armature coils. IEEE Transactions on Energy Conversion. 2007;22(3):621-628.

[3] Pinilla M, Martinez S. Selection of main design variables for low-speed permanent magnet machines devoted to renewable energy conversion. IEEE Transactions on Energy Conversion. 2011;26(3):940-945.

[4] Shin HJ, Choi JY, Park YS, Koo MM, Jang SM, Han H. Electromagnetic vibration analysis and measurements of double-sided axial-flux permanent magnet generator with slotless stator. IEEE transactions on magnetics. 2014;50(11):1-4.

[5] Ajily E, Ardebili M, Abbaszadeh K. Magnet defect and rotor eccentricity modeling in axial-flux permanentmagnet machines via 3-D field reconstruction method. IEEE Transactions on Energy Conversion. 2016;31(2):486-495.

[6] Mahmoudi A, Ping HW, Rahim NA. A comparison between the TORUS and AFIR axial-flux permanentmagnet machine using finite element analysis. In:2011 IEEE International Electric Machines \& Drives Conference (IEMDC). May 15, 2011. IEEE. p. 242-247. 
[7] Kahourzade S, Mahmoudi A, Ping HW, Uddin MN. A comprehensive review of axial-flux permanent-magnet machines. Canadian Journal of Electrical and Computer Engineering. 2014;37(1):19-33.

[8] Mahmoudi A, Rahim NA, Hew WP. Axial-flux permanent-magnet machine modeling, design, simulation, and analysis. Scientific Research and Essays. 2011;6(12):2525-2549.

[9] Min SG, Sarlioglu B. Analytical calculation of back EMF waveform for linear PM motors in slotted and slotless structures. IEEE Transactions on Magnetics. 2017;53(12):1-10.

[10] Zhu ZQ, Howe D, Bolte E, Ackermann B. Instantaneous magnetic field distribution in brushless permanent magnet DC motors. I. Open-circuit field. IEEE Transactions on Magnetics. 1993;29(1):124-135.

[11] Lubin T, Mezani S, Rezzoug A. Simple analytical expressions for the force and torque of axial magnetic couplings. IEEE Transactions on Energy Conversion. 2012;27(2):536-546.

[12] Ping J, Yue Y, Jin M, Fang S, Lin H, Hui Y, Ho SL. 3-D analytical magnetic field analysis of axial flux permanent-magnet machine. IEEE Transactions on Magnetics. 2014;50(11). Article Sequence Number: 8103504.

[13] Huang Y, Ge B, Dong J, Lin H, Zhu J, Guo Y. 3-D analytical modeling of no-load magnetic field of ironless axial flux permanent magnet machine. IEEE Transactions on Magnetics. 2012;48(11):2929-2932.

[14] Tiegna H, Amara Y, Barakat G. A new quasi-3-D analytical model of axial flux permanent magnet machines. IEEE Transactions on Magnetics. 2014;50(2):817-820.

[15] Egea A, Almandoz G, Poza J, Ugalde G, Escalada AJ. Axial-flux-machine modeling with the combination of FEM-2-D and analytical tools. IEEE Transactions on Industry Applications. 2012;48(4):1318-1326.

[16] Choi JY, Lee SH, Ko KJ, Jang SM. Improved analytical model for electromagnetic analysis of axial flux machines with double-sided permanent magnet rotor and coreless stator windings. IEEE Transactions on Magnetics. 2011;47(10):2760-2763.

[17] Sung SY, Jeong JH, Park YS, Choi JY, Jang SM. Improved analytical modeling of axial flux machine with a double-sided permanent magnet rotor and slotless stator based on an analytical method. IEEE Transactions on Magnetics. 2012;48(11):2945-2948.

[18] Koechli C, Perriard Y. Analytical model for slotless permanent magnet axial flux motors. In:2013 International Electric Machines \& Drives Conference. May 12, 2013. IEEE. p. 788-792.

[19]Furlani EP. Computing the field in permanent-magnet axial-field motors. IEEE Transactions on Magnetics. 1994;30(5):3660-3663.

[20] Chan TF, Lai LL, Xie S. Field computation for an axial flux permanent-magnet synchronous generator. IEEE Transactions on Energy Conversion. 2009;24(1):1-11.

[21] Azzouzi J, Barakat G, Dakyo B. Analytical modeling of an axial flux permanent magnet synchronous generator for wind energy application. In: 2005 IEEE International Conference on Electric Machines and Drives. May 15, 2005. IEEE. p. 1255-1260.

[22] Shin KH, Cho HW, Lee SH, Choi JY. Armature reaction field and inductance calculations for a permanent magnet linear synchronous machine based on subdomain model. IEEE Transactions on Magnetics. 2017;53(6). Article no. 8105804.

[23] Virtic P, Pisek P, Marcic T, Hadziselimovic M, Stumberger B. Analytical analysis of magnetic field and back electromotive force calculation of an axial-flux permanent magnet synchronous generator with coreless stator. IEEE Transactions on Magnetics. 2008;44(11):4333-4336.

[24] Boroujeni ST, Mohammadi AA, Oraee A, Oraee H. Approach for analytical modelling of axial-flux PM machines. IET Electric Power Applications. 2016;10(6):441-450.

(C) 2019 by the author(s). This work is licensed under a Creative Commons Attribution 4.0 International License (http://creativecommons.org/licenses/by/4.0/). Authors retain copyright of their work, with first publication rights granted to Tech Reviews Ltd. 